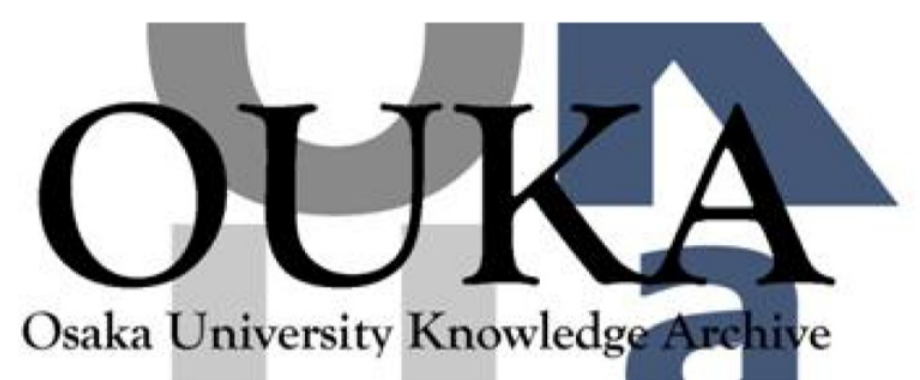

\begin{tabular}{|c|l|}
\hline Title & $\begin{array}{l}\text { Bragg reflection band width and optical } \\
\text { rotatory dispersion of cubic blue-phase Liquid } \\
\text { crystals }\end{array}$ \\
\hline Author(s) & $\begin{array}{l}\text { Yoshida, Hiroyuki; Anucha, Konkanok; Ogawa, } \\
\text { Yasuhiro et al. }\end{array}$ \\
\hline Citation & Physical Review E. 64(4) p. 042703-p.042703 \\
\hline Issue Date & $2016-10-21$ \\
\hline oaire:version & VoR \\
\hline URL & https://hdl.handle. net/11094/75690 \\
\hline rights & $\begin{array}{l}\text { Copyright (2016) by the American Physical } \\
\text { Society }\end{array}$ \\
\hline Note & \\
\hline
\end{tabular}

Osaka University Knowledge Archive : OUKA

https://ir. Library. osaka-u. ac. jp/

Osaka University 


\title{
Bragg reflection band width and optical rotatory dispersion of cubic blue-phase liquid crystals
}

\author{
Hiroyuki Yoshida, ${ }^{*}$ Konkanok Anucha, Yasuhiro Ogawa, Yuto Kawata, and Masanori Ozaki \\ Division of Electrical, Electronic and Information Engineering, Graduate School of Engineering, Osaka University, \\ 2-1 Yamada-oka, Suita, Osaka 565-0871, Japan \\ Jun-ichi Fukuda \\ Research Institute for Sustainable Chemistry, National Institute of Advanced Industrial Science and Technology (AIST), \\ 1-1-1 Higashi, Tsukuba 305-8565, Japan \\ Hirotsugu Kikuchi \\ Institute for Materials Chemistry and Engineering, Kyushu University, 6-1 Kasuga-koen, Kasuga, Fukuoka 816-8580, Japan
}

(Received 8 April 2016; published 21 October 2016)

\begin{abstract}
The Bragg reflection band width and optical rotatory dispersion of liquid crystalline cholesteric blue phases (BPs) I and II are compared by numerical simulations. Attention is paid to the wavelength regions for which the reflection bands with lowest photon energies appear, i.e., the [110] direction for BP I and the [100] direction for BP II. Finite difference time domain and $4 \times 4$ matrix calculations performed on the theoretical director tensor distribution of BPs with the same material parameters show that BP II, which has simple cubic symmetry, has a wider photonic band gap than BP I, which has body centered cubic symmetry, possibly due to the fact that the density of the double-twist cylinders in BP II are twice that in BP I. The theoretical results on the Bragg reflection band width are supported by reflectance measurements performed on BPs I and II for light incident along the [110] and [100] directions, respectively.
\end{abstract}

DOI: 10.1103/PhysRevE.94.042703

\section{INTRODUCTION}

Liquid crystalline (LC) cholesteric blue phases (BPs) typically appear between the cholesteric phase and the isotropic liquid in a chiral liquid crystal [1]. The submicron cubic orientational order exhibited by BPs I ( I $4{ }_{1} 32$ symmetry) and II $\left(P 4_{2} 32\right)$ make them attractive, both as subjects of soft matter physics and as candidate materials for next generation electro-optic and photonic applications [2-6]. The invention of the polymer-stabilization technology has substantially improved their stability, leading them to be considered as practical materials for displays and other tunable optical applications $[7,8]$.

One of the challenges for using BPs in optical applications is their low contrast ratio, i.e., light leakage between crossed polarizers. It is known that for propagating plane waves, BPs act like a cholesteric liquid crystal (ChLC), which has a one-dimensional helical structure $[9,10]$; therefore, the optical activity induced by the helical modulation of the optic axis reduces the contrast ratio in the vicinity of the Bragg reflection peaks. Recently, Liu et al. have measured the the optical rotatory power of polymer-stabilized BPs at three wavelengths and proposed that the contrast ratio can be enhanced by making slight adjustments in the analyzer angle [11]. They have explained the phenomenon by simulating the optical properties of randomly orientated twisted-nematic domains as an approximation for the structure of blue phases. On the other hand, we have previously shown by finite difference time domain (FDTD) calculations that the optical properties of BPs cannot be described correctly just by considering an array of double-twist cylinders, but rather, the distribution of the tensor

\footnotetext{
*yoshida@eei.eng.osaka-u.ac.jp
}

order parameter calculated from the Landau-de Gennes theory must be used [12-14]. It is likely that an analysis of the optical rotatory dispersion based on the tensorial order parameter distribution would provide a more in-depth understanding of the situation.

In this paper, we numerically investigate the optical properties of BPs I and II modeled from the Landau-de Gennes theory by FDTD and $4 \times 4$ matrix calculations [13-16]. We first present the photonic band structure of the two systems obtained from FDTD calculations and show the difference in the band gaps appearing in each system. We then focus on the band gap with the lowest energy and calculate the transmittance and optical rotatory dispersion for each BP. The $4 \times 4$ matrix calculations are performed on the dielectric tensor averaged on the plane normal to the propagation direction and are found to yield results close to those with three-dimensional FDTD calculations in wavelengths near the lowest energy gap. This is because light travels essentially as a plane wave in this region and, as a result, enables us to significantly reduce the computational load in predicting the amount of light leakage in BPs with various optical pitches. We find that BP II possesses a larger photonic band gap than BP I, possibly because of the larger density of the double-twist cylinders in the system and, consequently, larger light leakage between crossed polarizers for the same material parameters. In the final section of the paper, polarized reflection spectra of aligned BP samples are measured to corroborate the numerical findings.

\section{NUMERICAL SIMULATION}

\section{A. Methods}

The Landau-de Gennes theory, which describes the orientational order of the liquid crystal by a symmetric traceless 
tensor $\mathbf{Q}$ was used to describe the molecular orientation in BPs I and II. Since the technical details of calculating the order parameter tensor are described in detail elsewhere [17], we do not repeat them here; for this paper, the order parameter tensor distributions of the BP unit cells were calculated using the conditions described in Ref. [17] on a $64 \times 64 \times 64$ grid. The helicity of the system was right handed. The numerical calculations provide us with what is called the reduced order parameter tensor distribution; we normalized the order parameter tensor so that the maximum local isotropic order parameter $S_{\max }$ within the BP lattice is 0.7 , where $S^{2}=3 / 2 \operatorname{Tr}\left(\mathbf{Q}^{2}\right)[13,14,18]$. To simulate the optical properties of BPs, the order parameter tensor was converted to the dielectric tensor $\varepsilon_{i j}$ through the relation,

$$
\varepsilon_{i j}=\frac{2}{3} \Delta \varepsilon Q_{i j}+\varepsilon_{\mathrm{ave}} \delta_{i j},
$$

where $\Delta \varepsilon$ is the dielectric anisotropy of the host nematic, $\varepsilon_{\text {ave }}$ is the average dielectric permittivity, and $\delta_{i j}$ is Kronecker's $\delta . \Delta \varepsilon$ and $\varepsilon_{\text {ave }}$ are related to the ordinary and extraordinary refractive indices $n_{o}$ and $n_{e}$ according to the equation $\Delta \varepsilon=\left(n_{e}^{2}-n_{o}^{2}\right)$ and $\varepsilon_{\text {ave }}=\left(n_{e}^{2}+2 n_{o}^{2}\right) / 3$. We assumed values of $n_{e}=1.7$ and $n_{o}=1.5$ for our calculations.

A cholesteric liquid crystal, which has a sinusoidal distribution of the uniaxial director, was also modeled for comparison purposes. Assuming that the helix lies in the $z$ direction and has pitch $p$, the dielectric tensor is given by

$$
\varepsilon_{\mathrm{ChLC}}=\left[\begin{array}{ccc}
\frac{\varepsilon_{e}+\varepsilon_{o}}{2}+\frac{\Delta \varepsilon}{2} \cos 2 \phi & \frac{\Delta \varepsilon}{2} \sin 2 \phi & 0 \\
\frac{\Delta \varepsilon}{2} \sin 2 \phi & \frac{\varepsilon_{e}+\varepsilon_{o}}{2}+\frac{\Delta \varepsilon}{2} \sin 2 \phi & 0 \\
0 & 0 & \varepsilon_{o}
\end{array}\right] .
$$

Here, $\varepsilon_{e}=\varepsilon_{\mathrm{ave}}+2 \Delta \varepsilon S_{\max } / 3, \varepsilon_{o}=\varepsilon_{\mathrm{ave}}-\Delta \varepsilon S_{\max } / 3$, and $\phi=2 \pi z / p$. $\Delta \varepsilon$ was multiplied by $S_{\max }$ to purely compare the effect of the structure on the optical properties.

Two kinds of calculations were performed on the three LC phases. First, the photonic band structure was calculated by imposing Bloch's periodic boundary conditions in a unit cell [15]. The spatial and temporal discretizations were set so that $\Delta x=\Delta y=\Delta z$ and $\Delta t=0.565 \Delta x / c$ to ensure convergence [15]. Also, a one-dimensional calculation was performed for the cholesteric phase because the system is uniform on the plane perpendicular to the helical axis. The second set of calculations investigated the cross-polarized transmittance of a BP layer sandwiched between two glass substrates (with $n=1.6$ ) to directly compare the optical activity of BPs I and II in a standard device structure. Taking the substrate normal as the $x$ direction, the BP was assumed to have the [101] and [100] directions parallel to the $x$ axis for BPs I and II, respectively, and the [010] direction parallel to the $y$ axis. The incident light was assumed to have linear polarization along the $y$ axis. The pitch length and, consequently, the discretization steps were varied depending on the target wavelength of reflection.

\section{B. Photonic band structure}

Figure 1 shows the photonic band structure of BPs I and II. Reflecting the symmetry of each phase, the band structures are different: The lowest band gap appears in the $\Gamma-N$ direction in BP I and along the $\Gamma-X$ direction in BP II. Above the first
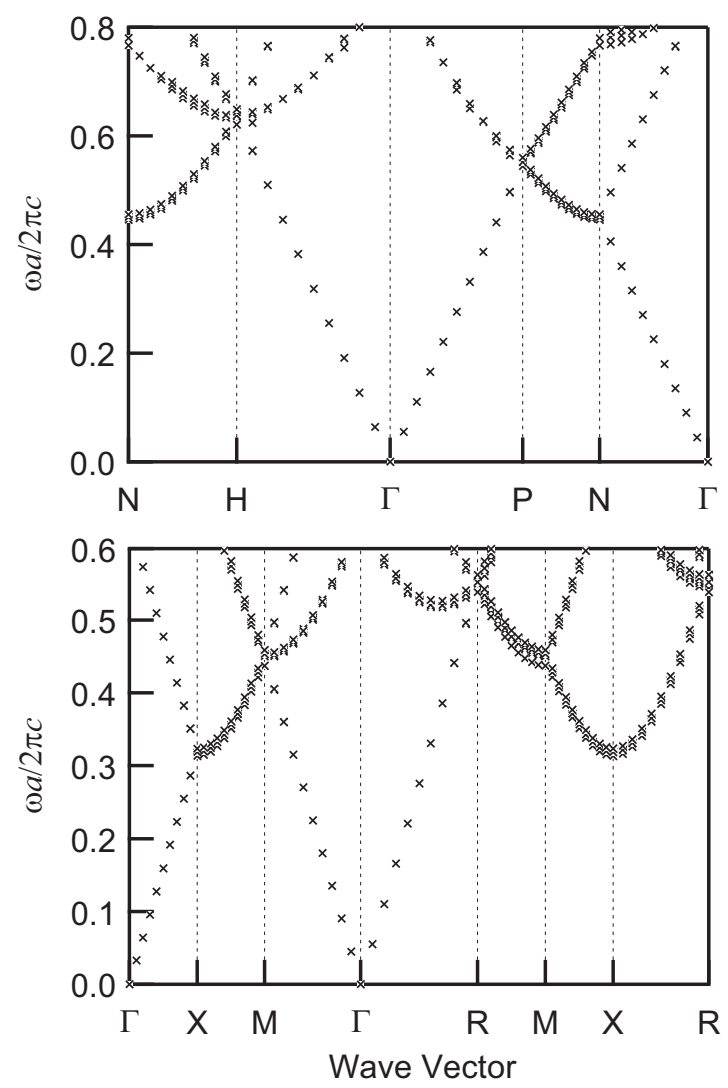

FIG. 1. Photonic band structure of BPs I and II.

stop band, the degeneracy of the eigenmodes breaks, indicating that the system is anisotropic. Therefore, depolarization effects can be avoided only when the wavelength of light is longer than that for the lowest stop band. Although polarizationindependent phase modulation has been proposed as one possible application of BPs [19,20], it should be noted that BPs are truly isotropic only in the limit $\lambda \rightarrow \infty$ [10]. This means that to remove the selective reflection peak from the operating wavelength of a BP device, such as a display or a lens without causing depolarization, BP materials with shorter pitches must be sought.

We now focus on the region in the Brillouin zone where the optical band gap with smallest energy appears. Figures 2(a)2 (c) show the photonic band structure along the $\Gamma-N$ direction for BP I and along the $\Gamma-X$ direction for BP II and the cholesteric phase near the Brillouin zone edge. Similar band structures are observed with a band-gap opening only for light with the same handedness as the helical structure [right circular polarization (RCP) for this case]. This corresponds to the so-called selective reflection band where circularly polarized light with the opposite handedness [left circular polarization (LCP)] is transmitted without being affected. The difference in the RCP and LCP eigenvalues is dispersive and reverses sign at the central frequency of the gap; this corresponds to anomalous optical rotatory dispersion, which is well known in the cholesteric phase [21]. The photonic band structure shows that the optical properties of cholesterics and BPs are qualitatively the same with the only major difference being the band-gap width. The cholesteric phase has the largest band 


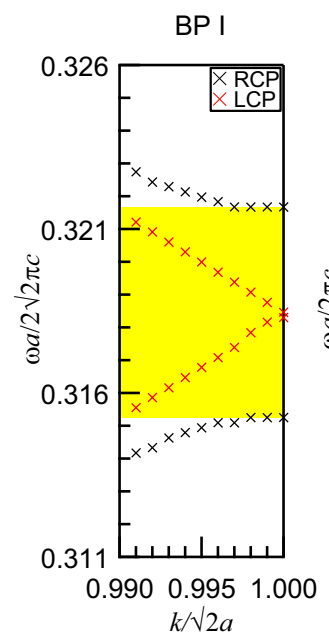

(a)

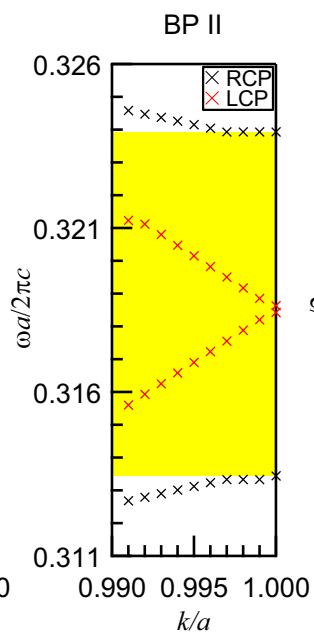

(b)

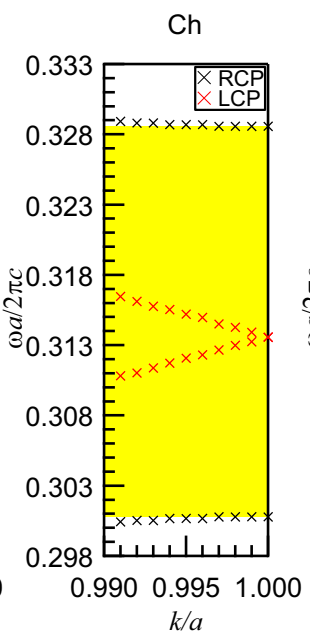

(c)

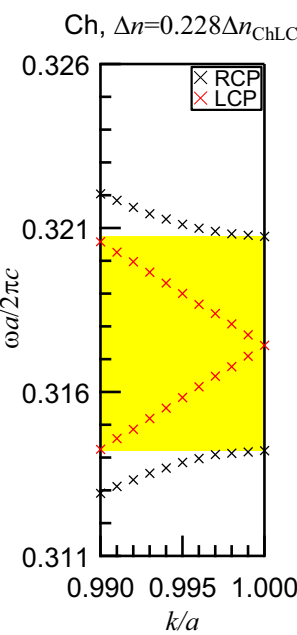

(d)

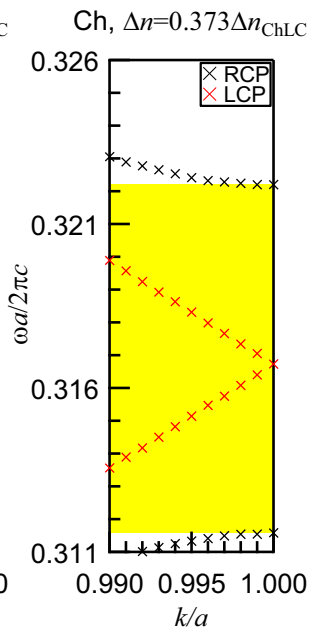

(e)

FIG. 2. Closeup of the photonic band structure near the reflection band with lowest energy in (a) BP I, (b) BP II, (c) the cholesteric phase, (d) the cholesteric phase with birefringence reduced to $22.8 \%$, and (e) the cholesteric phase with birefringence reduced to $33.7 \%$.

gap, followed by BP II and BP I, with relative stop-band widths $\Delta \omega / \omega_{c}=0.0202,0.0329$, and 0.0883 for BPs I, II, and the cholesteric phase, respectively.

The smaller band gaps observed in the BPs can be viewed as a trade-off for stretching the band gap to exist in three dimensions. Considering that the band-gap width of a cholesteric liquid crystal is given by $\Delta \lambda=\Delta n \times p$, an effective birefringence for the corresponding directions of each BP can be evaluated by comparing the bandgap widths: $\Delta n_{\mathrm{BPI}} / \Delta n_{\mathrm{ChLC}}=0.228$ and $\Delta n_{\mathrm{BPII}} / \Delta n_{\mathrm{ChLC}}=$ 0.373. As shown in Figs. 2(d) and 2(e), almost identical photonic band structures are obtained when the birefringence of the ChLC is reduced to $22.8 \%$ and $37.3 \%$ of the original value with $\Delta \omega / \omega_{c}=0.0206$ and 0.0335 , respectively. This again supports the similarity in the optical behavior in the BPs and cholesterics. The slight shift in band-gap frequency corresponds to the difference in the average refractive indices between BPs and ChLCs: The average refractive index of the BPs is the isotropic average $\sqrt{\left(n_{e}^{2}+2 n_{o}^{2}\right) / 3}$ whereas that for the cholesteric phase is $\sqrt{\left(n_{e}^{2}+n_{o}^{2}\right) / 2}$.

Hornreich and co-workers had calculated the photonic band structures of BPs by the plane wave expansion method as early as 1993 [22]. The main focus of their report, however, was on whether a complete photonic band gap would open (which is difficult with realistic material parameters), and the relative widths of the reflection band had not been discussed. On the other hand, there is an early experimental study by Marcus that discussed the difference between BPs and cholesterics by measuring the band width of reflection from the three phases confined in thick $(<0.5-\mathrm{mm})$ cells [9]: The results of our numerical analyses are close to the values obtained in this experiment (values of $\Delta \lambda / \lambda$ are approximately $0.013,0.019$, and 0.054 in BPs I, II, and the cholesteric phase, respectively, yielding effective birefringence values of $24 \%$ and $35 \%$ ). A possible explanation for BP II showing a larger band gap than BP I could be that the density of the double-twist cylinders in BP II is twice as large as that in BP I. Since the doubletwist cylinders contribute to the chirality of the system, the modulation in the refractive index becomes larger in BP II, leading to a larger photonic band gap.

\section{Transmittance and optical rotatory dispersion}

Figure 3 shows the transmittance and polarization spectra for BPs I and II with $p=288.72$ and $408.32 \mathrm{~nm}$ sandwiched between glass substrates. The pitch was chosen so that the band gap would appear near $640 \mathrm{~nm}$ for both BPs, and 10-pitch-length-thick BP layers (corresponding to 10 and 20 unit cells for BPs I and II, respectively) were assumed. For the wavelengths near the lowest-energy band gaps, transmittance calculations of three-dimensional FDTD and $4 \times 4$ matrix calculations using the dielectric tensor distribution averaged on the plane perpendicular to the direction of propagation yield close results. This is because near the lowest-energy band gaps, the wavelength of light is comparable to the period of the structure and only experiences an averaged dielectric tensor on the plane normal to the direction of propagation. Figure 4 shows the FDTD results of electric field amplitudes of light transmitted through the BP, averaged on the plane normal to light propagation. The minimum and maximum electric field amplitudes within the plane, which are also plotted, fall within $\pm 0.02 \mathrm{~V} / \mathrm{m}$ from the average amplitude, implying that the output light is a quasiplane wave.

The applicability of the $4 \times 4$ matrix method to BPs has been shown by Berreman who used a simplified BP model derived from Landau theory (along with two other models comprising only uniaxial or isotropic order parameter tensors) [23] and Bohley and Scharf who used a model in which cylindrical structures made from twisting uniaixal molecules (doubletwist cylinders) were floating in an isotropic medium [24]. Our results show that $4 \times 4$ matrix calculations provide reliable results also for BP models calculated numerically.

The fact that a one-dimensional calculation is sufficient greatly simplifies the simulation of devices. As an example, light leakage (cross-polarized transmittance) of $10-\mu \mathrm{m}$-thick BPs I and II with $\langle 110\rangle$ and $\langle 100\rangle$ orientations are calculated 


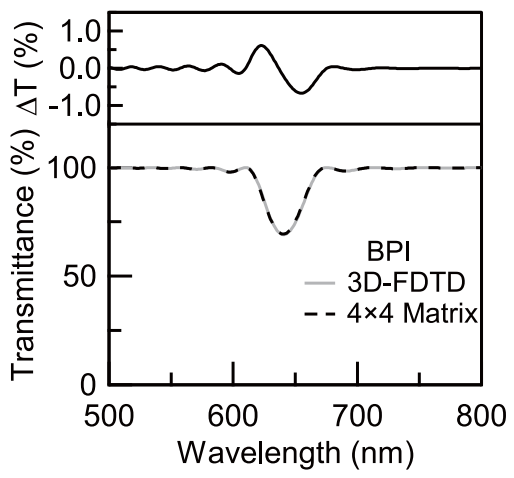

(a)

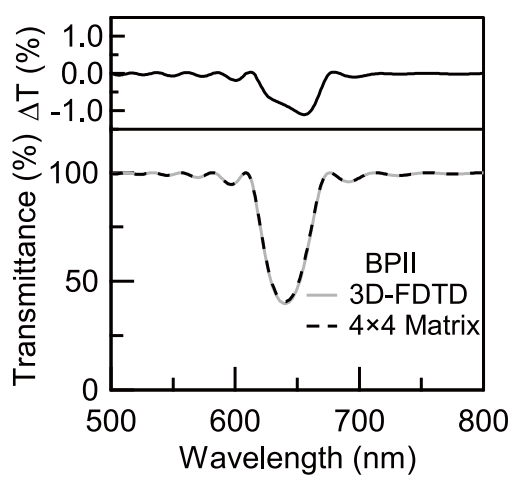

(b)

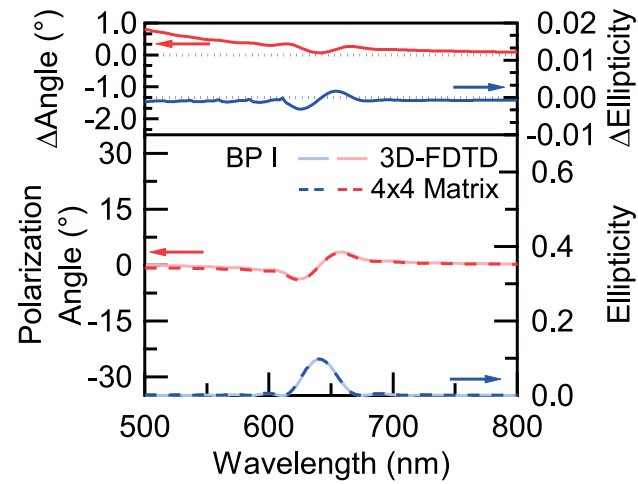

(c)

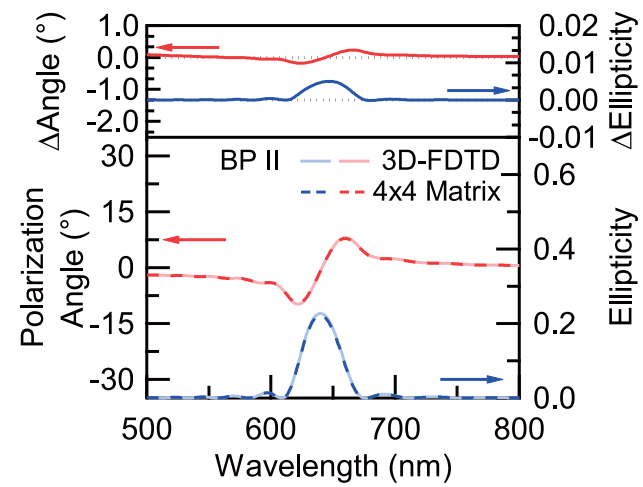

(d)

FIG. 3. (a) Transmittance and (b) polarization spectra of BPs I and II along the [101] and [100] directions by the FDTD assuming a threedimensional dielectric tensor distribution for the BPs (3D-FDTD) and Berreman's $4 \times 4$ matrix formulation using the dielectric tensor distribution averaged on the plane perpendicular to the direction of polarization [23].

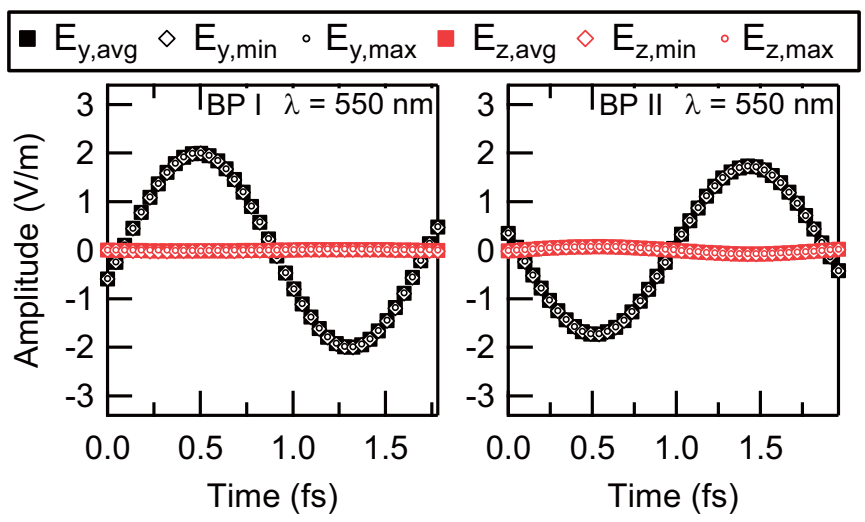

(a)
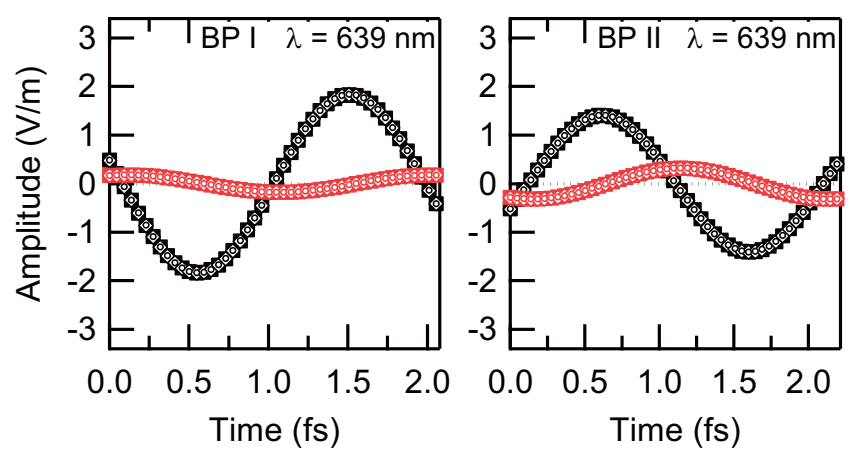

(b)
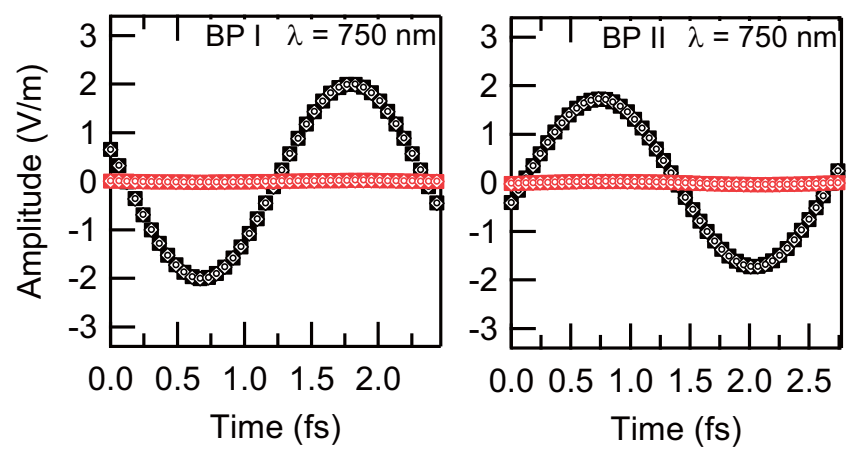

(c)

FIG. 4. Electric field amplitudes of light transmitted through BPs I and II at wavelengths of (a) $550 \mathrm{~nm}$, (b) $639 \mathrm{~nm}$, and (c) $750 \mathrm{~nm}$, averaged on the plane normal to the propagation direction. The maximum and minimum values within the plane are also plotted to show the plane wavelike nature of light propagation in the BP.

at a wavelength of $400 \mathrm{~nm}$ for different lattice constants and maximum order parameters. As long as the Bragg wavelength is shorter than $400 \mathrm{~nm}$, the light leakage is smaller at longer wavelengths, therefore, this calculation can be used as a guide to estimate the maximum light leakage. The results are presented in Fig. 5. As the figure shows, the cross-polarized transmittance increases as the pitch or the birefringence increases because of increased optical activity. Also, in agreement with the photonic band structure calculations, BP II shows greater light leakage because of the larger optical activity. These calculations can therefore be used as a guide to select material or device parameters that fulfill the specifications required for a particular application. 


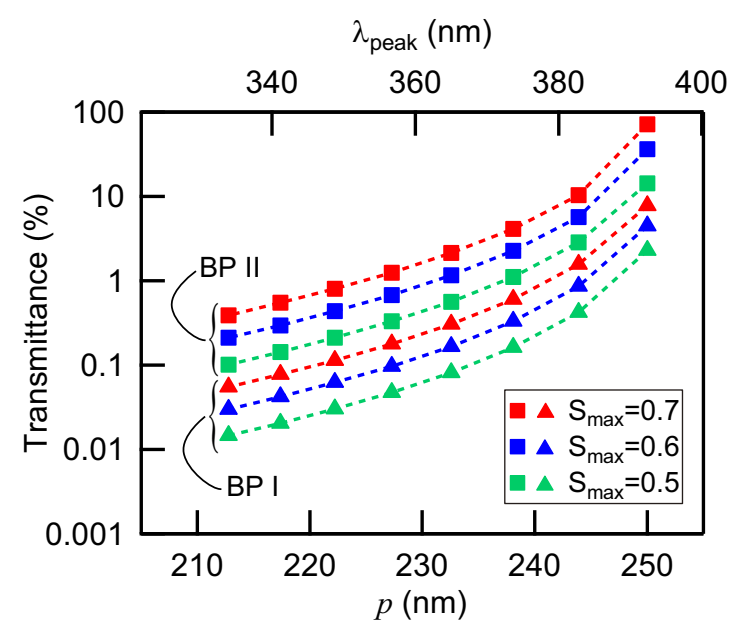

FIG. 5. Simulated transmittance through ideal crossed polarizers for light at $\lambda=400 \mathrm{~nm}$ propagating along the $\langle 110\rangle$ and $\langle 100\rangle$ directions of BPs I and II, respectively. The horizontal axis shows the period of BPs I and II along the $\langle 110\rangle$ and $\langle 100\rangle$ directions, respectively, and refractive indices of $n_{e}=1.7, n_{o}=1.5$ are assumed with different values of $S_{\max }$. The vertical axis has a logarithmic scale to emphasize the difference in cross-polarized transmittance.

We note that Dolganov et al. have investigated the applicability of the Kramers-Kronig equations to the circular dichroism and optical rotation of ChLCs [25]. Because of the similarity in the dielectric distribution of BPs and ChLCs, application of the Kramers-Kronig equations to BPs yields similar results where the difference between the experimental and the calculated values increases at shorter wavelengths due to the increased contribution of adiabatic polarization rotation (polarization rotation through a twisted birefringent material in the so-called Mauguin regime [26]). Because BPs show different band gaps along different directions, it should be of interest in the future to investigate circular dichroism and optical rotation for propagation along different crystal axes.

\section{EXPERIMENTAL SECTION}

Experiments were performed to confirm the theoretical prediction that BP II has a wider band gap than BP I. The reflection spectrum of a monodomain $\mathrm{BP}$ sample was measured as the sample changed phase from BP I to BP II. As stated previously, Marcus has performed quantitative comparisons of the relative band widths of BPs I and II by measuring the reflection from a thick cell (but with an unknown exact cell gap) [9]. On the other hand, Barbet-Massin and Pierański have measured the reflectance from a monodomain BP I and discussed its order parameter [27,28]. However, measurements had not been made on a monodomain BP II. Here, we (i) used a cell with known thickness $(8.85 \mu \mathrm{m})$ and (ii) confirmed the orientation of the platelets by Kossel diagram observations to precisely compare the reflectance between BPs I and II. We also performed $4 \times 4$ matrix simulations to quantitatively compare the reflectance of the two phases.

The BP sample was prepared by doping a chiral dopant [ISO-(6OBA $)_{2}, 6.5 \mathrm{wt} \%$ ] in a nematic LC mixture $(1 \times 1$ mixture of $5 \mathrm{CB}$ and JC-1041XX, JNC Corporation). The

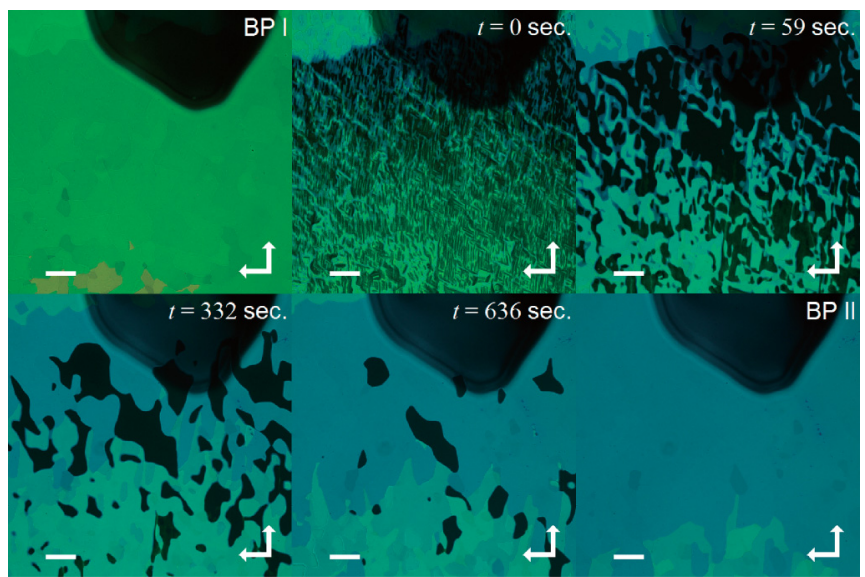

(a)

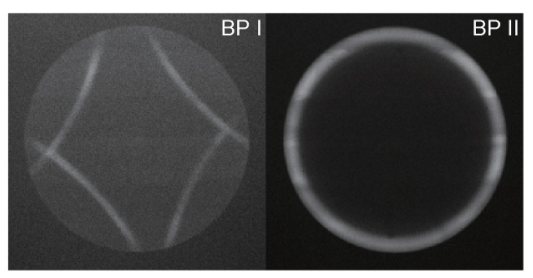

(b)

FIG. 6. (a) Polarized optical microscope image of a sample as the temperature is raised slowly to induce a transition from BP I to BP II. The length of the scale bar is $100 \mu \mathrm{m}$, and the arrows indicated the direction of the polarizers. (b) Kossel diagrams observed for BPs I and II using a probe wavelength of $436 \mathrm{~nm}$.

phase sequence of the sample determined from polarized optical microscopy was approximately cholesteric $\left(45.6^{\circ} \mathrm{C}\right) / \mathrm{BP}$ I $\left(46.7^{\circ} \mathrm{C}\right) / \mathrm{BP}$ II $\left(47.7^{\circ} \mathrm{C}\right) /$ isotropic using a commercial hot stage (Linkam, LTS-350). The sample was infiltrated in a glass sandwich cell assembled from two substrates with a unidirectional rubbing treatment. The sample was first cooled from the isotropic phase to BP I and then heated and cooled several times within the BP I temperature range so that the whole field of view was covered with BP crystals with a $\langle 110\rangle$ axis along the cell normal. The sample was then slowly heated to the BP II temperature range to induce a phase transition. The polarized reflectance spectra were measured using a fiber-optic spectrometer (Ocean Optics, USB-4000) equipped with a multimode fiber (core diameter of $550 \mu \mathrm{m})$ and coupled to a microscopic optical system with $10 \times$ magnification. The probe light, polarized linearly by a wire-grid polarizer, was incident on the sample from normalcy (through the objective lens) after being reflected by a metallic half-mirror. The reflected light was detected through the half-mirror after passing through a polarizer, which had its transmission axis set perpendicular to the incident polarizer, and a lens with a focal length of $200 \mathrm{~mm}$.

Figure 6 shows polarized microscopy images of the sample at the BP I-II phase transition. The black marks at the top of the images are guides indicating the position of the sample. In the BP I temperature, a uniform green texture was observed [Fig. 6(a), first figure], which was found from Kossel diagram observations [Fig. 6(b)] to correspond to BP I with the $\langle 110\rangle$ 


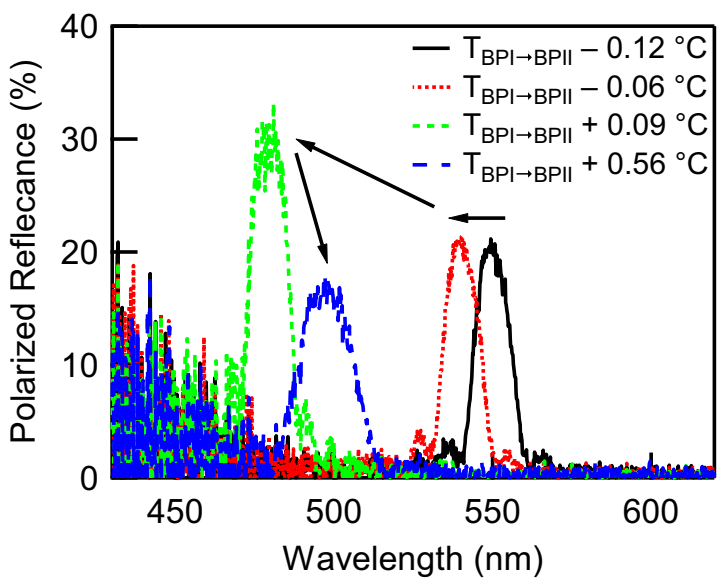

FIG. 7. Polarized reflectance spectra of the sample as the temperature is raised slowly to induce a transition from BP I to BP II. $T_{\mathrm{BP} \text { I } \rightarrow \text { BP II }}$ indicates the BP I to BP II transition temperature. Arrows are drawn as guides to show how the reflectance spectrum evolves.

direction in the viewing direction. As the temperature was raised, a sudden change occurred in the texture with blue and black domains appearing where only green domains existed. The black domains gradually disappeared whereas the blue domains grew larger, and after some time, a uniform blue texture was obtained [Fig. 6(a), final figure]. In some parts, the color changed from green to blue without the appearance of black textures (e.g., top left part of the figures). The lattice plane giving the blue texture was confirmed from Kossel diagram observations to be a $\{100\}$ plane of BP II [Fig. 6(b)]. The BP lattice undergoes a rotation by $45^{\circ}$ upon changing phase from BP I to BP II $[29,30]$.

Figure 7 shows the polarized reflectance spectra of the sample near the point of transition (measurements were made after a uniform texture was obtained). The most obvious feature of the phase transition is the discontinuous shift in the peak reflection wavelength $\left(\lambda_{\text {peak }}\right)$ from approximately 540.2 to $479.4 \mathrm{~nm}$. Using the refractive index of the host nematic LC available from literature [31] (Table I) and the relationship $d_{h k l}=a / \sqrt{h^{2}+k^{2}+l^{2}}$ (where the lattice constant $a$ is equal to the pitch $p$ in BP I and $2 a=p$ in BP II), the pitch is estimated to be $240.6 \mathrm{~nm}$ in BP I and $299.6 \mathrm{~nm}$ in BP II. The helical pitch therefore elongates discontinuously at the BP I $\rightarrow$ BP II phase transition. Although the precise mechanism requires further investigation, this discontinuous change in pitch at the phase transition could be one of the

TABLE I. Parameters used to simulate the reflection spectra in Fig. 7.

\begin{tabular}{lcc}
\hline \hline Peak wavelength $(\mathrm{nm})$ & 540.2 & 479.4 \\
$n_{e}$ & 1.70431 & 1.72375 \\
$n_{o}$ & 1.52621 & 1.53456 \\
$p(\mathrm{~nm})$ & 240.6 & 299.6 \\
Lattice constant $(\mathrm{nm})$ & 240.6 & 149.8 \\
Lattice number & 26 & 59 \\
Order parameter distribution & BP I & BP II \\
$S_{\max }$ & 0.38 & 0.26 \\
\hline \hline
\end{tabular}

reasons why the lattice rotates from $\langle 110\rangle$ along the cell normal in BP I to $\langle 100\rangle$ in BP II. At the phase transition, the period along the $\langle 110\rangle$ direction increases from $\sqrt{2} \times p=340.3$ to $423.7 \mathrm{~nm}$, but because the cell thickness is constant, the BP must unwind to reduce the number of lattices present. On the other hand, if the lattice changes its orientation to $\langle 100\rangle$, the period becomes $299.6 \mathrm{~nm}$, which is shorter than the original period along $\langle 110\rangle$. Therefore, by rotating the lattice, the BP can avoid the energy cost of having to unwind the helical structure and reduce the lattice number.

Another observation we can make from the reflectance spectra is the discrete shift in the reflection wavelength in BP I from approximately 550.0 to $540.2 \mathrm{~nm}$. Such a discrete shift in the reflection wavelength has been observed in cholesteric liquid crystals with strong surface anchoring, corresponding to the winding or unwinding of the helix in half-pitch intervals [32]. Although we did not see them in our sample, the appearance of Grandjean steps, which are indicators of the discrete change in molecular rotation number, has been reported in BPs confined in a wedge cell [33]. Therefore, we also infer that the discontinuous shift in wavelength is due to a discrete change in the number of lattices existing in the cell. The reflection peak shift corresponds to a change in pitch from 240.6 to $246.7 \mathrm{~nm}$ using average refractive indices 1.5878 and 1.5862 at the two wavelengths, respectively [31]. Dividing the cell-gap $(8.85 \mu \mathrm{m})$ by the pitch length yields approximately 26 and 25.5 as the number of periods $(N)$, implying that similar to the cholesteric helix, the BP I lattice also winds or unwinds in half-integer steps. An interesting question to consider is at which position within the lattice the BP is anchored to the substrate. Although this is out of scope of this paper, an in-depth analysis of the order parameter distribution may provide insight on the molecular configuration of a $\mathrm{BP}$ in contact with a rubbed substrate.

As the temperature is increased in BP II, the reflection peak shows a discrete redshift and reduction in peak reflectance, attributed to an elongation in pitch and reduction in birefringence. However, broadening of the peak also occurs, which contradicts the fact that a smaller birefringence should result in a narrower reflection band. This implies that, as the temperature approaches the isotropic clearing point, the effective thickness of the BP decreases, possibly due to the temperature gradient along the cell-depth direction. Although this does not affect the findings of this paper where we are focusing on the BP I $\rightarrow$ BP II transition, this calls for the need to take extra care in suppressing temperature gradients in the sample when comparing the spectra near the clearing point.

Since the refractive index, pitch, and the number of periods existing within the cell are known, quantitative discussions on the reflectance are now possible by numerical calculations. The $4 \times 4$ matrix calculations were performed to simulate the experimentally obtained spectra using the parameters summarized in Table I. The wavelength of reflection is determined primarily by the refractive indices and the lattice constant, and other parameters, including the order parameter distribution, contribute to the reflectance. $S_{\max }$ is the maximum order parameter within the BP lattice and is used as an adjusting parameter to yield the best matching spectrum. To confirm the difference between BPs I and II, the reflectance 


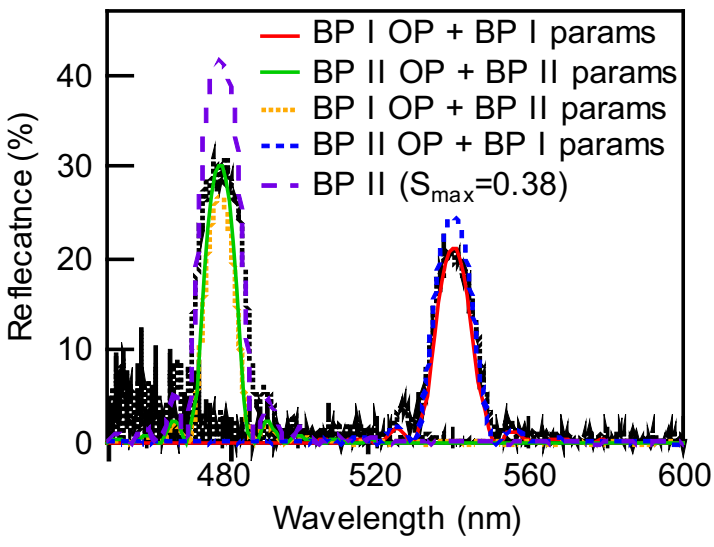

FIG. 8. Simulated polarized reflectance spectra calculated using the combination of numerical parameters summarized in Table I and the order parameter distributions for BPs I and II. OP in the annotation refers to "order parameter." The dotted curves are experimental reflectance spectra, corresponding to temperatures $T_{\text {iso }}-0.06^{\circ} \mathrm{C}$ and $T_{\text {iso }}+0.09^{\circ} \mathrm{C}$ in Fig. 7.

spectra were calculated also for the case when the numerical parameters for the two peaks $\left(n_{e}, n_{o}\right.$, lattice constant, and lattice number), and the order parameter distributions for the two BPs (including $S_{\max }$ ) were interchanged. Figure 8 shows the results of numerical calculations; good agreement is obtained between theory and experiment using the parameters shown in Table I. When the BP I dielectric tensor distribution was assumed, but fitting parameters for the peak at $490.2 \mathrm{~nm}$ were used (orange broken line in Fig. 8), the reflectance became lower than that obtained experimentally. On the other hand, when the parameters for the peak at $540.2 \mathrm{~nm}$ were used with the dielectric tensor distribution of BP II, the reflectance exceeded the value obtained experimentally (blue broken line in Fig. 8). This implies that the increase in reflectance observed at the phase transition from BP I $\rightarrow$ BP II simply is not only due to a change in refractive index and number of periods, but also due to BP II intrinsically possessing a larger photonic band gap. It should be noted here, however, that the value of $S_{\max }$ is 0.38 for BP I and 0.26 for BP II. If we assume $S_{\max }=0.38$ for BP II, the reflectance becomes much higher than that obtained in experiment (purple line in Fig. 8). One possible interpretation of this is that the local maximum order parameter decreases at the BP I-BP II transition as a result of the rearrangement of the double-twist cylinders and shortening of pitch.

\section{CONCLUSIONS}

The optical properties of BPs I and II were investigated by numerical analyses and experiment. The photonic band structures of BPs show that BPs are only isotropic in the limit $\lambda \rightarrow \infty$ and that they show optical rotation with a reduced effective birefringence, compared to the one-dimensional cholesteric liquid crystal. Among the two cubic BPs, BP II has a larger photonic band gap and thus larger reflectance and light leakage possibly owing to the higher density of double-twist cylinders in the unit cell. To select material parameters that would fulfill the requirements of a particular device, simulations should first be performed to estimate the depolarization occurring in the device. To this end, as long as light travels as a plane wave, that is, as light propagates along the [110] and [100] directions of BPs I and II, respectively, one-dimensional $4 \times 4$ matrix calculations can replace three-dimensional differential calculations, thereby greatly simplifying the process of searching for desirable material parameters.

\section{ACKNOWLEDGMENTS}

This work was supported by JSPS KAKENHI Grants No. 23107519, No. 23656221, No. 24656015, and No. 16K13862. The authors thank JNC Corporation for kindly providing the nematic LC.
[1] D. C. Wright and N. D. Mermin, Rev. Mod. Phys. 61, 385 (1989).

[2] W. Cao, A. Muñoz, P. Palffy-Muhoray, and B. Taheri, Nat. Mater. 1, 111 (2002).

[3] M. Ravnik, G. P. Alexander, J. M. Yeomans, and S. Žumer, Proc. Natl. Acad. Sci. U.S.A. 108, 5188 (2011).

[4] J.-i. Fukuda and S. Žumer, Nat. Commun. 2, 246 (2011).

[5] A. Tiribocchi, G. Gonnella, D. Marenduzzo, E. Orlandini, and F. Salvadore, Phys. Rev. Lett. 107, 237803 (2011).

[6] H. Yoshida, K. Inoue, H. Kubo, and M. Ozaki, Opt. Mater. Express 3, 842 (2013).

[7] H. Kikuchi, M. Yokota, Y. Hisakado, H. Yang, and T. Kajiyama, Nat. Mater. 1, 64 (2002).

[8] J. Yan and S.-T. Wu, Opt. Mater. Express 1, 1527 (2011).

[9] M. Marcus, Phys. Rev. A 25, 2276 (1982).

[10] V. A. Belyakov, E. I. Demikhov, V. E. Dmitrienko, and V. K. Dolganov, J. Exp. Theor. Phys. 62, 1173 (1985).

[11] Y. Liu, Y.-f. Lan, H. Zhang, R. Zhu, D. Xu, C.-Y. Tsai, J.-K. Lu, N. Sugiura, Y.-C. Lin, and S.-T. Wu, Appl. Phys. Lett. 102, 131102 (2013).
[12] M. Ojima, Y. Ogawa, R. Ozaki, H. Moritake, H. Yoshida, A. Fujii, and M. Ozaki, Appl. Phys. Express 3, 032001 (2010).

[13] Y. Ogawa, J.-i. Fukuda, H. Yoshida, and M. Ozaki, Opt. Lett. 38, 3380 (2013).

[14] Y. Ogawa, J.-i. Fukuda, H. Yoshida, and M. Ozaki, Opt. Express 22, 3766 (2014).

[15] A. Taflove, Computational Electrodynamics: The FiniteDifference Time-Domain Method, 3rd ed. (Artech House, Boston, 2005).

[16] D. W. Berreman, J. Opt. Soc. Am. 62, 502 (1972).

[17] J.-i. Fukuda, M. Yoneya, and H. Yokoyama, Phys. Rev. E 80, 031706 (2009).

[18] S. Urban, B. Gestblom, W. Kuczyński, S. Pawlus, and A. Würflinger, Phys. Chem. Chem. Phys. 5, 924 (2003).

[19] Y.-H. Lin, H.-S. Chen, H.-C. Lin, Y.-S. Tsou, H.-K. Hsu, and W.-Y. Li, Appl. Phys. Lett. 96, 113505 (2010).

[20] S. Yabu, Y. Tanaka, K. Tagashira, H. Yoshida, A. Fujii, H. Kikuchi, and M. Ozaki, Opt. Lett. 36, 3578 (2011). 
[21] P. G. D. Gennes and J. Prost, The Physics of Liquid Crystals, 2nd ed. (Oxford University Press, Oxford, 1995).

[22] R. M. Hornreich, S. Shtrikman, and C. Sommers, Phys. Rev. E 47, 2067 (1993).

[23] D. W. Berreman, in Liquid Crystals and Ordered Fluids, edited by A. C. Griffin and J. F. Johnson (Plenum, New York, 1984), p. 925.

[24] C. Bohley and T. Scharf, Opt. Laser Eng. 43, 329 (2005).

[25] P. V. Dolganov, G. S. Ksyonz, V. E. Dmitrienko, and V. K. Dolganov, Phys. Rev. E 87, 032506 (2013).

[26] P. Yeh and C. Gu, Optics of Liquid Crystal Displays, 2nd ed. (Wiley, Hoboken, NJ, 2009).
[27] R. Barbet-Massin and P. Pierański, J. Phys., Colloq. 46, C3 (1985).

[28] R. Barbet-Massin and P. Pierański, J. Phys. Lett. 45, 799 (1984).

[29] H. Stegemeyer, T. Blumel, K. Hiltrop, H. Onusseit, and F. Porsch, Liq. Cryst. 1, 3 (1986).

[30] B. Pansu, J. Phys. II France 5, 573 (1995).

[31] J. B. Lias, H. Kikuchi, M. Kimura, and T. Akahane, Jpn. J. Appl. Phys. 50, 081607 (2011).

[32] K. Funamoto, M. Ozaki, and K. Yoshino, Jpn. J. Appl. Phys. 42, L1523 (2003).

[33] W. Kuczynski and H. Stegemeyer, Naturwissenschaften 67, 310 (1980). 